\title{
Maternal responsiveness of outdoor sows from first to fourth parities
}

\author{
Suzanne Held ${ }^{\mathrm{a}, *}$, Georgia Mason ${ }^{\mathrm{b}}$, Michael Mendl ${ }^{\mathrm{a}}$ \\ ${ }^{\text {a }}$ Centre for Behavioural Biology, Department of Clinical Veterinary Science, \\ University of Bristol, Langford BS40 5DU, UK \\ ${ }^{\mathrm{b}}$ Department of Animal \& Poultry Science, University of Guelph, \\ Guelph, Ont., Canada N1G 2W1
}

Accepted 13 September 2005

Available online 17 October 2005

\begin{abstract}
Sows of indoor breeds are well known to differ in the speed and strength with which they respond to a piglet trapped under their bodies. There is also evidence from two previous studies that a sow's responsiveness to playback of a simulated trapped piglet scream is related to the number of crushed piglets in her litter. This raises the possibility of using responsiveness in the Piglet Scream Test as a measure of mothering ability in commercial pigs. In outdoor sows, piglet losses due to crushing are thought to be even higher than in indoor sows, yet comparatively little is known about the maternal responsiveness in these animals, or about its relationship to piglet mortality. The present study therefore investigated the behaviour of outdoor sows in the Piglet Scream Test and a piglet handling test. Its main aims were to investigate changes and individual consistency in test responses over several parities, and the relationship between a sow's behaviour in these tests and the survival of her piglets under commercial outdoor production conditions. Data were collected for first to fourth parities on 41 Landrace/Duroc $\times$ Large White sows kept in individual farrowing paddocks. Data from second and third parities had to be combined because of data losses incurred by severe floods in 2000 and the UK Foot and Mouth Epidemic of 2001. Tests of maternal responsiveness comprised a Piglet Scream Test on Day 5 or 6 post-partum and a test of the sows' responses to handling of their piglets on Days 1, 9 or 10, and 24 or 25 (Piglet Defence Test). Comparisons within parities revealed that sows responded more strongly and faster to playback of a piglet scream than of a control sound (bird call). Large individual differences were evident in responses in both tests, and there was some evidence for consistency in individual differences across parities. The responses to playback of the
\end{abstract}

\footnotetext{
* Corresponding author. Tel.: +44 117 9289571; fax: +44 1179289582.
}

E-mail address: suzanne.held@bris.ac.uk (S. Held). 
piglet scream and control sound were strongly correlated and declined from first to fourth parities, as did the sows' defensiveness scores. Responses in the two tests were correlated in the sows' first, but not subsequent parities. There was no evidence for an association between the test responses of the sows and total or live-born mortality levels in their litters which gives some first indication of a potentially limited usefulness of responses in the Piglet Scream Test as measures of mothering ability, at least in outdoor sows on commercial units.

(C) 2005 Elsevier B.V. All rights reserved.

Keywords: Outdoor pigs; Maternal responsiveness; Piglet Scream Test; Consistency; Parities

\section{Introduction}

During lactation, most deaths of live-born piglets are caused by the sow 'crushing' or 'overlying' piglets which are trapped by her body (Svendsen et al., 1986; Riart et al., 2000, cited in Edwards, 2002). Piglets are trapped by the sows' lying behaviours and posture changes (e.g. Weary et al., 1996a; Herskin et al., 1998; Marchant et al., 2001; Vieuille et al., 2003), but not all trapped piglets die. Weary et al. (1996a) showed that few trapped piglets are killed outright through traumatic injury. The likelihood of dying as a result of trapping seemed to depend largely on how long piglets were trapped for (Weary et al., 1996a). This, in turn, is partly determined by their vitality (Weary et al., 1996b), but also possibly by how the sow responds when she has trapped a piglet.

It has long been known that sows differ in their responses to a squealing piglet and in how likely they are to sit or stand up in response to screams of a trapped piglet (Signoret et al., 1975; Cronin and Cropley, 1991; Hutson et al., 1991). How strongly and quickly a sow reacts to playback of a pre-recorded piglet scream are thus widely used measures of maternal responsiveness in pigs. The idea of this Playback or 'Piglet Scream' Test is to simulate a potential overlay situation (Cronin and Cropley, 1991). A study on indoor sows kept in a family farrowing pen by Wechsler and Hegglin (1997) provided some first evidence that the responsiveness of a sow in this Piglet Scream Test may indeed be related to the survival rate of her piglets: sows that were more responsive in the test crushed fewer piglets than less responsive sows. Wechsler and Hegglin's study (1997) thus suggested that responsiveness to a piglet scream might be a good measure of mothering ability, where mothering ability is the ability of a sow to enhance the fitness and survival of her offspring through her behaviour (Fleming et al., 1996).

Since then, several studies have shown large individual differences in responses in the Piglet Scream Test, with the authors suggesting potential underlying genetic variation in maternal responsiveness (e.g. Špinka et al., 2000; Pitts et al., 2002; Grandinson et al., 2003). A genetic component, it is argued, would open up the possibility of selecting for mothering ability (e.g. Cronin and Cropley, 1991) and thus of reducing piglet mortality by enhancing the maternal characteristics of breeding sows (see Edwards, 2002). However, since Wechsler and Hegglin's original study (1997), two further studies, which investigated the link between responses in the Piglet Scream Test and piglet mortality, failed to find evidence for a phenotypic relationship, either under experimental (Špinka et al., 2000) or commercial conditions (Grandinson et al., 2003), while Andersen et al.'s recent study does 
report a relationship in sows kept on a University farm (Andersen et al., 2005). It remains uncertain, therefore, whether a link between maternal responsiveness and piglet mortality does indeed exist on commercial farms, where an arguably greater variety of factors affect the survival of piglets in the immediate post-partum period than under experimental conditions (see Edwards, 2002 for recent review). Furthermore, most studies to date have looked at sows in only one or at most two parities. It is thus not clear whether individual differences remain consistent across parities. Consistency of individual differences across parities would strengthen the case for maternal responsiveness being a stable individual characteristic with a possible underlying genetic component.

This study therefore investigated the responses of outdoor sows in two tests of maternal responsiveness across parities 1-4. Its main aims were to study the development of and individual consistency in test responsiveness, and to look at the relationship between a sow's test responses and the survival of her piglets under commercial outdoor production conditions. Comparatively little is known about the development of maternal behaviour and its relationship to piglet mortality in outdoor pigs. In outdoor sows, piglet losses due to overlying are thought to be even higher than in indoor sows, because the piglets are not as protected from the body movements of their mother (Edwards et al., 1994; Riart et al., 2000, cited in Edwards, 2002). As in indoor sows, not all trapped piglets die (Vieuille et al., 2003). This raises the possibility that in outdoor pigs, too, differences in maternal responsiveness may contribute to differences in pre-weaning piglet survival.

We addressed the following specific questions: (i) can the standard Piglet Scream Test of maternal responsiveness be applied in commercial outdoor conditions; (ii) do different test measures of maternal responsiveness correlate; (iii) does maternal responsiveness as tested change with increasing age or parity; (iv) are sows consistent relative to each other in their test responsiveness over several parities; and finally (v) is there evidence in outdoor sows kept under commercial conditions for a link between maternal responsiveness as tested and piglet survival?

We adapted the standard Piglet Scream Test originally developed by Hutson et al. (1991) for commercial outdoor conditions. As Wechsler and Hegglin (1997), we included as a control the playback of a sound thought to be biologically irrelevant to sows in order to validate our test. As a new potential measure of maternal responsiveness in outdoor sows we also assessed the sows' reaction to their piglets being weighed and handled in the farrowing hut in early, middle and late lactation, while the sows were being fed in the farrowing paddock.

\section{Methods}

\subsection{Animals and housing}

This study was carried out between March 2000 and April 2002 at Downland Pigs, a commercial outdoor pig unit in Wiltshire, UK. Data were collected for first (gilts) to fourth parities from 41 sows (Landrace/Duroc $\times$ Large White). All sows were mated using Large White boars or Large White semen, and kept in groups of no more than six in paddocks during gestation. One week before their estimated farrowing date, sows were introduced to 
individual farrowing paddocks $(15 \mathrm{~m} \times 20 \mathrm{~m})$, each containing a floorless, blunt-top Aframe hut ('Rawnsley Arc'; $190 \mathrm{~cm} W \times 205 \mathrm{~cm} L \times 123 \mathrm{~cm} \mathrm{H}$ ) and water trough. Huts were bedded with straw and made of plywood with galvanised metal roofs, which were insulated with a layer of polystyrene. The entrance to each hut could be closed off with a wooden door, and an inspection and ventilation window was located at the back wall. A further inspection hole was cut into the roof in the back left corner (approximately $35 \mathrm{~cm} \times 25 \mathrm{~cm}$ ). This was large enough to push the tape recorder through for the Piglet Scream Test and see the sow inside at the same time without being seen. After inspections or tests the hole was covered up again with galvanised metal sheeting.

\subsection{Management}

Sows were fed a standard commercial diet in the form of sow pellets once a day in the morning. On the first full day of their life (D1), piglets were tooth-clipped and tail-docked by farm staff as part of the farm's standard practice at the time of the study, and each piglet was weighed in a bucket suspended from spring scales and 'handled' afterwards by seizing it round the chest with both hands and holding it up for approximately $15 \mathrm{~s}$. Piglets were weighed and 'handled' again on D9/10, and on D24/25 before weaning. They were always weighed inside the hut in the mornings when the sow was receiving her daily feed ration in the paddock. Piglets were cross-fostered within the first $48 \mathrm{~h}$ after birth where appropriate to keep litter sizes as even and close to 10 piglets as possible, which was part of the farm's standard procedure. They were not provided with creep feed, but able to follow their mothers out of the hut and eat her feed. Piglets were weaned on the first Wednesday or Thursday after they were 24 days old.

\subsection{Tests of maternal responsiveness}

\subsubsection{Responsiveness to playback of a piglet scream (Piglet Scream Test)}

At the start of the study, a 30 s recording was made of the screams of a 6-day-old piglet (Sony Cassette Recorder TCM-939). The piglet was seized round the chest and gently restrained on the ground. As a control stimulus we used a $30 \mathrm{~s}$ recording of a nightingale call (see also Wechsler and Hegglin, 1997). The volume control of the tape recorder was calibrated using a sound level meter (Tenung 72-6604) to ensure that the piglet scream and bird call were played back at the same volume. Playback volume for the piglet scream was 64-84 dB at $1.5 \mathrm{~m}$ from the tape recorder, which was equivalent to the sound level measured at $1.5 \mathrm{~m}$ distance from the screaming piglet during recording (cf. Hutson et al., 1993); the volume of the bird call at playback was $64-80 \mathrm{~dB}$ at $1.5 \mathrm{~m}$ from the tape recorder.

Piglet Scream Tests were carried out on Day 5 or 6 in the mornings after feeding when the sow had returned to the hut. D5/6 was chosen in agreement with the farmer in order to avoid agitating the sows in the first few days after farrowing. When the sow was lying down but neither asleep nor nursing, the tape recorder was introduced into the hut through the inspection hole in the roof and held approximately $80 \mathrm{~cm}$ from the ground for quick retrieval without the sows seeing or hearing it. The piglet call or control sound was then played for $30 \mathrm{~s}$, and the sow's latency to her first behavioural response measured. Her 
Table 1

Response scores in the playback test and their definitions (modified from Hutson et al., 1993)

\begin{tabular}{ll}
\hline Score & Sow's strongest behavioural response \\
\hline 0 & No reaction \\
1 & Head movement \\
2 & Head movement towards sound \\
3 & Body movement towards sound \\
4 & Sits up \\
5 & Stands up \\
6 & Stands up and orients towards loudspeaker \\
7 & Gentle physical contact with loudspeaker \\
8 & Nudges or paws loudspeaker \\
\hline
\end{tabular}

strongest behavioural response during the 30 s playback period was also scored, using the categories in Table 1. Each sow was tested three times with each sound, alternating between them. Half the sows were tested with the piglet scream first, half with the control sound first. A new playback commenced when the sow had settled down and lay down after the previous one. The interval between subsequent sounds never exceeded 20 min.

\subsubsection{Response to piglet handling-'Piglet Defence Test'}

The second test assessed the sows' behaviour when their piglets were handled. The idea of the test was to simulate a potential threat to the sow's litter. In the morning, the sow was offered her daily food ration in the paddock at least $10 \mathrm{~m}$ from the hut as usual. After she started feeding, a handler was locked into the hut with the piglets for weighing, and 'handling' as described in Section 2.2. The sow was then scored according to her behaviour: continue feeding in paddock or be near hut. Behavioural scoring was done by a second person, who also guarded the hut entrance. The potential threat was thus signalled to the sow by the presence of a person by the hut entrance and the calls of her handled piglets. The sow's behaviour was recorded for every 'handled' piglet in her litter. Recording thus continued until all her piglets had been handled. Defensive behaviour was then expressed as the percentage of 'handled' piglets for which the sow was by the hut. Most, but not all, piglets vocalized during handling. We therefore had to rule out piglet vocalization as a potential confound before further analyses of defensiveness. Regression analyses showed no consistent relationship between piglet vocalizations and defensiveness scores, but in order to completely remove any possible confounding effects we partialled out any variation in defensiveness due to piglet vocalizations before the mortality analyses. To investigate whether the sows' defensiveness changed over the course of lactation, we tested them on D1, D9/10 and D24/25.

\subsection{Mortality data}

Because of the transport restrictions during and after the Foot and Mouth Epidemic, it was impossible to remove sufficient numbers of dead piglets from the farm for post mortem examination as originally planned. We therefore had to use two measures of pre-weaning mortality in this study: total mortality, calculated as the total number of piglets born per sow minus the number of piglets fostered off minus the number of own weaned piglets; 
live-born mortality calculated as the total number born per sow minus the number of stillborn minus the number of piglets fostered off minus the number of own weaned piglets. Total mortality was expressed as a percentage of total number born, live-born mortality as a percentage of number of live-born piglets. In the morning of D1, dead piglets were removed from the litter and classified on the farm as 'still' or 'live'-born according to the state of the hoof periople, the extent of membrane cover of the nasal and oral cavities and the presence of birth defects or mummification (after Wells, 1978).

\subsection{Data analysis}

Data were collected on 41 sows. However, severe flooding in 2000 and the UK Foot and Mouth Disease epidemic and its aftermath in 2001 resulted in data losses in all four parities, but particularly in Parities 2 and 3. For the analyses, we therefore combined Parities 2 and 3 into a mixed data set containing data from 27 of the 41 sows in their second parity and 14 sows in their third. To investigate the effect of parity on maternal responsiveness, and individual consistency across parities, we included in the respective analyses only sows, for which data were available for three parities (first, second or third and fourth). These were 12 sows for the Piglet Scream Test, and 33 sows for the Piglet Defence Test.

The following variables were used in the analyses: response strength in each separate Piglet Scream Test (maximum response score, see Table 1); total response strength in Piglet Scream Tests (sum of maximum scores in the three repeats); latency to first response in each separate Piglet Scream Test (in seconds; ceiling value of $30 \mathrm{~s}$ ); total latency to first response in Piglet Scream Tests (in seconds; sum of latencies in the three repeats; ceiling value of $90 \mathrm{~s}$ ); defensiveness (as percentage, see Section 2.3.2); total and live-born preweaning mortality (as percentage of total born, see Section 2.4).

Data were analysed using Minitab (Minitab ${ }^{\circledR}$, Release 13) and SPSS (SPSS ${ }^{\circledR}$, Version 11). Relative consistency within parities was assessed using Pearson's or Spearman's Rank Correlations. Consistency across parities was also investigated with Pearson and Spearman's Rank Order Correlations. Non-normally distributed data were square-root transformed. Where transformation did not result in normal or nearly normal distributions, we used nonparametric statistics. All comparisons of two group means or medians are two-tailed.

The relationship between the sows' responses in the Piglet Scream and Defence Tests using data from all three parity groups was analysed in a General Linear Model (GLM) with Piglet Scream Test responses as the response variables, and defensiveness as the explanatory variable blocked for parity with sow as a random factor. Standardized residuals were checked for normality. The relationship between the two tests of maternal responsiveness and piglet mortality was analysed in separate GLMs with mortality measures as the response variables, litter size as a covariate and test responses as the explanatory variables. In the corresponding analyses using data from all parities, piglet mortality was also blocked for parity with sow as a random factor. Correlation analyses found responses to the piglet scream to be correlated to responses to the bird call. We therefore repeated the analyses above after having partialled out responsiveness to the bird call on the assumption that this reflects the sows' general, baseline reactivity to external stimuli. 
Figures display standard deviations rather than standard errors of the mean to indicate variability between individuals (Grafen and Hails, 2002). Further details for the statistical tests are given with the respective results.

\section{Results}

\subsection{Response to piglet scream and control sound in the Piglet Scream Test}

Frequency distributions of the sows' total response strengths in the three parities are given in Fig. 1. Sows responded significantly stronger to the piglet scream than to the bird call in all three parities (Fig. 2a; Paired $t$-Tests; Parity $1: N=34, T=7.06, p<0.001$; Parity 2/3: $N=28, T=5.33, p<0.001$; Parity 4: $N=21, T=4.06, p=0.001)$. Total response latencies are given in Fig. 2b. Sows responded faster to the piglet scream than to the bird (control) sound in all three parities (Wilcoxon Matched Rank Tests; Parity 1: $N=34$, $W=5.0, p<0.001$; Parity 2/3: $N=28, W=20.0, p<0.001$; Parity $4: N=21, W=17.5$, $p=0.003$ ). Individual variation was large in both measures as indicated by the standard deviation bars in Fig. 2.

Sows, which responded the strongest to the piglet scream, also responded the fastest in all three parities (Spearman's Rank Correlations; Parity 1: $N=34, r_{\mathrm{S}}=-0.71, p<0.001$; Parity 2/3: $N=28, r_{\mathrm{S}}=-0.620, p<0.001$; Parity 4: $\left.N=21, r_{\mathrm{S}}=-0.651, p=0.001\right)$.

Finally, the response strengths and latencies to the piglet scream were correlated to responses to the bird call in all three parities (total response strength-Parity 1: $N=34$, $r=0.631, p<0.001$; Parity 2/3: $N=28, r=0.753, p<0.001$; Parity $4: N=21, r=0.784$,

$p<0.001$; total response latency-Parity 1: $N=34, r=0.726, p<0.001$; Parity $2 / 3$ : $N=28, r=0.807, p<0.001$; Parity 4: $N=21, r=0.624, p=0.003)$.

\subsection{Effect of repeated testing within parities on responses in the Piglet Scream and Piglet Defence Tests}

\subsubsection{Piglet Scream Tests}

Responses were correlated between first and last repeats of the piglet scream (Repeat 1 versus Repeat 3; Parity 1: $N=34, r=0.707, p<0.001$; Parity 2/3: $N=26, r=0.758$, $p<0.001$; Parity 4, $N=22, r=0.684, p<0.001)$. Responses to the control sound were also correlated over repeated testing within parities (Repeat 1 versus Repeat 3; Parity 1: $N=34, r=0.348, p=0.04$; Parity $2 / 3: N=26, r=0.684, p<0.001$; Parity $4: N=22$, $r=0.857, p<0.001)$.

How strongly sows responded to the piglet scream decreased significantly over the three consecutive tests in each parity (Repeated Measures Analysis; Parity 1: $F_{2,66}=25.23$, $p<0.001$; Parity 2/3: $F_{2,52}=10.64, p<0.001$; Parity $\left.4: F_{2,42}=9.18, p<0.001\right)$. In contrast, their responses to the control sound decreased significantly with repeated testing only in the second/third parity, and tended to in the first (Repeated Measures Analysis; Parity 1: $F_{2,66}=2.74, p=0.072$; Parity $2 / 3: F_{2,52}=16.80, p<0.001$; Parity 4: $\left.F_{2,42}=1.99, p=0.15\right)$. 
Response latencies to the piglet scream increased over the three repeats in the sows' first and second/third parities, and tended to increase in Parity 4 (Friedman's Analysis of Variance of Ranks (Repeated Measures) tests; Parity 1: $N=34$, Chi-square $=12.95$,
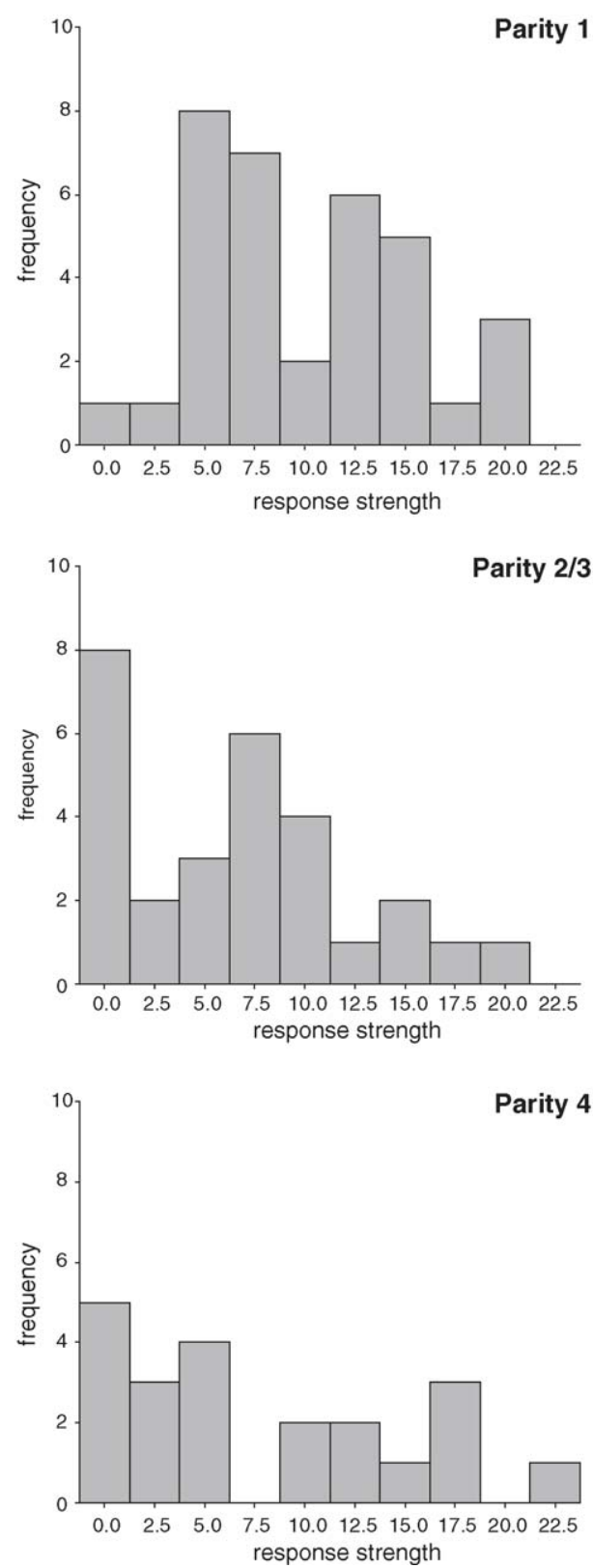

Fig. 1. Frequency distribution of total response strengths in the Piglet Scream Test. 

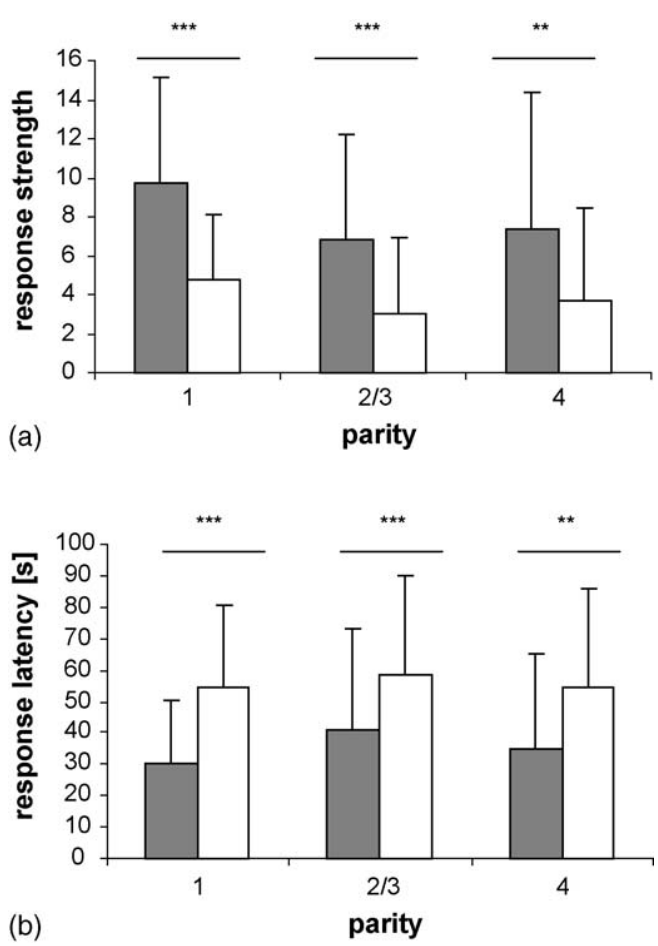

Fig. 2. Total response strength and latency to three playbacks of a piglet scream (dark bars) and control sound (light bars) in three parities; shown are means \pm S.D.s; $(* * *)$ denotes a significant difference at $p<0.001 ;(* *)$ at $p<0.01$.

d.f. $=2, p=0.002$; Parity 2/3: $N=27$, Chi-square $=10.29$, d.f. $=2, p=0.006$; Parity 4: $N=22$, Chi-square $=5.58, p=0.061$ ). Latency to respond to the control sound increased significantly in the first parity and tended to increase in the combined second/third (Friedman's Analysis of Variance of Ranks (Repeated Measures) tests; Parity 1: $N=34$, Chi-square $=7.22$, d.f. $=2, p=0.027$; Parity 2/3: $N=27$, Chi-square $=5.37$, d.f. $=2$, $p=0.068$; Parity 4: $N=22$, Chi-square $=0.26, p=0.876$ ).

\subsubsection{Piglet Defence Test}

The sows' defensive response to their piglets' being handled was tested on D1, D9/10 and D24/25 of each lactation. Median values decreased from D1 to D24/25 in gilts (first parity), but this trend was not significant because of large differences between individuals (Friedman's Analysis of Variance of Ranks (Repeated Measures) tests; Parity 1: $N=32$, Chi-square $=1.64$, d.f. $=2, p=0.441$ ). In Parity $2 / 3$ defensiveness increased significantly on D24/25 $(N=33$, Chi-square $=7.51$, d.f. $=2, p=0.023)$. In Parity 4, defensiveness was generally low and did not change during lactation $(N=19$, Chi-square $=1.56$, d.f. $=2$, $p=0.459$ ).

In the first parity, a sow's responsiveness in the Piglet Defence Test on D1 was correlated to her responsiveness on D9/10 and D24/25 (Spearman's Rank Correlation; $N=32$, D1 
versus D9/10: $r_{\mathrm{S}}=0.531, p=0.001 ; \mathrm{D} 1$ versus $\mathrm{D} 24 / 25, r_{\mathrm{S}}=0.445, p=0.011 ; \mathrm{D} 9 / 10$ versus $\left.\mathrm{D} 24 / 25, r_{\mathrm{S}}=0.142, p=0.439\right)$. In the second/third parity, overall responsiveness had declined, with more sows not responding at all, but responsiveness on D1 still tended to be correlated to responsiveness on D24/25 (Spearman's Rank Correlation; $N=33$, D1 versus D9/10: $r_{\mathrm{S}}=0.129, p=0.475 ; \mathrm{D} 1$ versus $\mathrm{D} 24 / 25, r_{\mathrm{S}}=0.460, p=0.071 ; \mathrm{D} 9 / 10$ versus $\mathrm{D} 24 / 25, r_{\mathrm{S}}=0.100, p=0.580$ ). Finally, in the fourth parity, no significant relationship emerged between the responsiveness ranks of sows on D1, D9/10 and D24/25 (Spearman's Rank Correlations; $N=19$ ) because of the large number of ties: $47.4 \%$ of sows ( 9 of the 19 , for which data existed for all 3 days) showed no response in the Piglet Defence Test on any of the 3 test days.

\subsection{Effect of parity on responsiveness of individual mothers}

\subsubsection{Changes over parities}

3.3.1.1. Piglet Scream Test. Twelve sows were tested in all three parities. Response strengths to the piglet scream decreased significantly between first and fourth parities (Fig. 3a; Repeated Measures Analysis: $F_{2,22}=3.55, p=0.046$ ). How strongly the sows responded to the control sound was also affected by parity, with the strongest responses in the first parity (Fig. 3b; Repeated Measures Analysis: $F_{2,22}=5.043, p=0.016$ ).
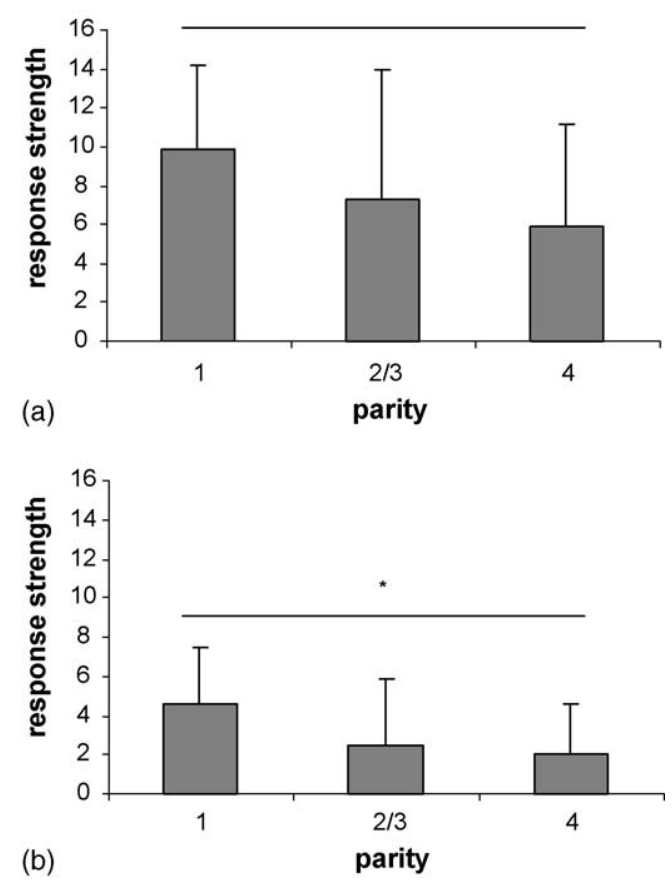

Fig. 3. Total response strength to three playbacks of a piglet scream (a) and control sound (b) in three parities; given are means \pm S.D. for the same 12 sows in each parity; $(*)$ denotes a significant parity effect at $p<0.05$. 


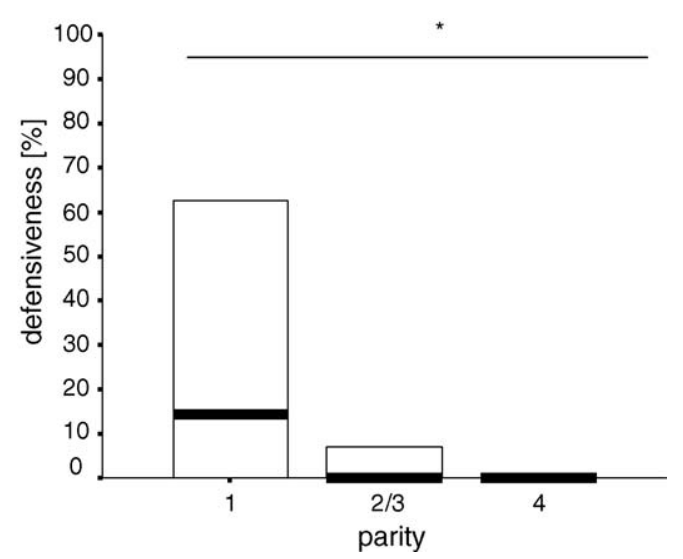

Fig. 4. Boxplot of defensiveness scores on Day 1 of lactation in three parities; given are medians (fat lines), first and third quartiles; $(*)$ denotes a significant parity effect at $p<0.05$.

Changes in latency to respond to the piglet scream and control sound were nonsignificant.

3.3.1.2. Piglet Defence Test. Defensiveness scores on D1 of lactation are shown in Fig. 4. The sows' responsiveness in the Piglet Defence Test (D1) declined significantly over the three parities (Friedman's Analysis of Variance of Ranks (Repeated Measures) tests; $N=33$, Chi-square $=6.37$, d.f. $=2, p=0.041$ ).

\subsubsection{Individual consistency across parities}

3.3.2.1. Piglet Scream Tests. Twelve sows were tested in all three of their parities. From the first to the second/third parity, sows remained consistent relative to each other in their response strength (Pearson's Correlation: $N=12, r=0.682, p=0.015)$. First and fourth parities, and second/third and fourth parities also tended to be correlated $(N=12 ; \mathrm{P} 1$ and P4: $r=0.521, p=0.08 ; \mathrm{P} 2 / 3$ and P4: $r=0.534, p=0.074$ ).

There was no evidence for consistency across parities in latency to respond (P1 and P2/ $3, r=-0.208, p=0.517 ; \mathrm{P} 1$ and $\mathrm{P} 4: r=-0.467, p=0.174 ; \mathrm{P} 2 / 3$ and $\mathrm{P} 4: r=0.205$, $p=0.570)$.

3.3.2.2. Piglet Defence Test. Thirty-three sows were tested on D1 in all three of their parities. Sows remained consistent in their defensiveness rankings between their second/ third and fourth parities (Spearman Rank Correlations; P1 and P2/3: $N=33, r_{\mathrm{S}}=0.227$, $p=0.204 ; \mathrm{P} 2 / 3$ and $\mathrm{P} 4: N=33, r_{\mathrm{S}}=0.438, p=0.011 ; \mathrm{P} 1$ and $\mathrm{P} 4: N=33, r_{\mathrm{S}}=0.0 .249$, $p=0.162)$.

\subsection{Relationship between responses in the Piglet Scream and Piglet Defence Tests}

For each of the three parities, we tested whether the sows' total response strength in the Piglet Scream Test was related to their behaviour in the Piglet Defence Test on D1. Initially, analyses only included the 11 sows, for which we had a complete set of responses for both 
Table 2

Mortality in three parities; given are averages \pm S.E.M.s

\begin{tabular}{llll}
\hline & Parity 1 & Parity 2/3 & Parity 4 \\
\hline Live-born mortality (\%) & $12.95 \pm 2.10$ & $13.34 \pm 2.47$ & $12.03 \pm 1.70$ \\
Total mortality (\%) & $14.04 \pm 2.18$ & $13.60 \pm 2.27$ & $12.54 \pm 1.67$ \\
\hline
\end{tabular}

tests. No significant relationships emerged between playback responses and defensiveness scores in any of the parities $(N=11$, Parity $1: r=0.478, p=0.137$; Parity $2 / 3: r=-0.033$, $p=0.923$; Parity 4: $r=-0.097, p=0.776)$. We then analysed separately for each parity data from all sows with records in the respective parity so as to increase replicate numbers. In these analyses, a significant correlation between the sows' responses in the two tests emerged in Parity 1, but not in the following parities (Parity 1: $N=29, r=0.399, p=0.032$; Parity 2/3: $N=26, r=0.012, p=0.955$; Parity $4: N=20, r=0.069, p=0.772$ ). How long it took sows to respond to the piglet scream was not related to their responses in the defence test in any parity (Parity 1: $N=31, r=-0.130, p=0.485$; Parity $2 / 3: N=26, r=-0.001$, $p=0.995$; Parity 4: $N=20, r=-0.070, p=0.770$ ).

Across parities, no significant relationship was found between the sows' total response strength in the Piglet Scream Tests and their response in the defence tests $\left(F_{1,19}=0.44\right.$, $p=0.513$ ), nor between their response latency in the Piglet Scream Tests and their defensiveness $\left(F_{1,24}=2.17, p=0.154\right)$.

\subsection{Relationship between maternal responsiveness and piglet mortality}

\subsubsection{Piglet Scream Test}

Mortality data are shown in Table 2. Repeated Measures ANOVA did not reveal any significant differences between parities (live-born mortality: $F_{2,68}=0.125, p=0.883$; total mortality: $\left.F_{2,68}=0.173, p=0.841\right)$.

There was no evidence for a relationship between total response strength or latency and piglet mortality in any parity. Partialling out baseline responsiveness (to the control sound) increased the amount of variation in mortality explained by responses to the piglet scream in gilts (first litters), but the effect remained non-significant.

Across parities, no significant relationship emerged (total response strength and total mortality: $F_{1,38}=0.27, p=0.604$; total response strength and live-born mortality: $F_{1,38}=0.36, p=0.551$; total response latency and total mortality: $F_{1,38}=0.35, p=0.556$; total response latency and live-born mortality: $F_{1,38}=0.10, p=0.759$ ). Partialling out underlying variation in responses to the control sound did not significantly change the relationship (total response strength and total mortality: $F_{1,38}=0.39, p=0.534$; total response strength and live-born mortality: $F_{1,38}=0.51, p=0.48$; total response latency and total mortality: $F_{1,38}=0.16, p=0.695$, total response latency and live-born mortality: $\left.F_{1,38}=0.37, p=0.545\right)$.

\subsubsection{Piglet Defence Test}

The sow's responses to their piglets' being handled on D1 were not related to total or live-born mortality in any parity. There was also no evidence for a relationship between the sows' defensiveness scores on D1 and piglet mortality across parities. 


\section{Discussion}

The main result of this study is that the responsiveness of outdoor sows in two tests of maternal behaviour declined from first to fourth parities. Within parities, sows differed from each other in how strongly they reacted in the tests, and there was also some evidence that individuals were consistent relative to each other across parities, that is sows that were the strongest responders remained the strongest even if absolute responsiveness levels declined. Sows responded more strongly and faster to playback of a piglet scream than of a control sound, and responses to the two sounds were strongly correlated in all parities. The animals habituated to playback of the piglet scream after triple testing within a maximum of $100 \mathrm{~min}$ in all three parities, and their responses were correlated across the three repeats. The study found no indication of an association between the sows' test responses and mortality levels in their litters.

A few studies on one or a maximum of two litters per sow have previously also reported a decline with parity in responsiveness to vocalizations of a trapped or squeezed piglet. These studies focussed on indoor systems and genotypes. Hutson et al. (1992), for example, tested the behavioural responses of Large White sows of varying age in farrowing crates to the playback of a piglet distress call. Sows were either in their first, second, fourth or sixth parity, and Hutson et al. (1992) reported faster, but not stronger, responses in gilts (first parity) than in multiparous sows. Thodberg et al.'s study (2002) indicated quicker responses to playback of a screaming piglet by Landrace $\times$ Yorkshire sows in their first than in their second parity. In Vieuille et al.'s study (2003), six sows of an indoor breed (Large White $\times$ Landrace) were kept in outdoor farrowing huts and studied over their first two parities. Vieuille et al. (2003) observed that the sows seemed to be more responsive to squeals of piglets trapped under their bodies in their first parities than in their second parities. Our findings expand these previous results to outdoor sows and to their fourth parity. The decreasing responsiveness in our and previous studies could be specific to threat situations to piglets only. It is clearly adaptive for sows in the early post-partum period to be sensitive to auditory stimuli coming from the piglets so as to prevent crushing, as tested in the Piglet Scream Test. Increased sensitivity to other sounds could also help the piglets' survival during this time, for example, through early detection of predators which would complement other anti-predator behaviours described for feral and wild pigs (Graves, 1984). In that case, responses to control sounds in Piglet Scream Tests generally could also be measures of the sow's responsiveness to a perceived threat to her piglets. Declining responses to both the piglet scream and the control sound could thus indicate a specific decline in maternal responsiveness with age or increasing parity.

Alternatively, the sows' declining responses could signify a general waning of reactivity to external stimuli in multiparous sows caused by age-related factors, such as increasing habituation to external stimulation or increasing deafness. This would be on the assumption that the control sound was of little biological significance to the sows. Responsiveness to the control sound can then be interpreted as a baseline measure of general reactivity during the early post-partum period. In our study, responses to the piglet scream and control sound not only differed in magnitude but were also correlated, and both decreased between first and fourth parities. This suggests as an alternative explanation a decline of general 
reactivity with increasing age, parity and maternal experience, rather than just a specific decline in maternal responsiveness to infant cues.

Finally, it is possible that the decline in maternal responsiveness over the four parities could indicate changes in parental 'input' or 'effort' with increasing parity or age as predicted under certain conditions by Parental Investment Theory (Evans, 1990). Parental input is related to parental 'investment', but needs to be distinguished from it (CluttonBrock, 1984; Evans, 1990). Parental investment after Trivers (1972) is any investment by the parent in its offspring that increases the offspring's survival chances but at a reproductive cost to the parent, that is at a cost to the parent's ability to invest in future offspring. In contrast, parental input refers simply to the care or resources that the parent provides to the offspring regardless of the reproductive costs this incurs (Evans, 1990). Thus, what we report on here are measures of parental (maternal) input, rather than measures of parental investment, since we have no record of the actual reproductive costs to the sows of providing for the piglets in the three parities. The predictions of Parental Investment Theory for changes in parental input with age are much less clear than predictions for parental investment. Parental input is predicted to either (a) decrease or (b) increase with age, depending on its relationship with parental investment (Clutton-Brock, 1984; Evans, 1990).

(a) If there is evidence for a strong positive correlation between parental investment and input, then input can be used as an indicator of investment (Evans, 1990). If this were to be the case in sows, then our observed decrease in parental input would go against the prediction from Parental Investment Theory that parental investment should increase with age in iteroparous species, such as the pig, in which reproductive value declines with age (Terminal Investment Hypothesis, Williams, 1966). However, parental investment is notoriously difficult to assess because of the difficulties of accurately measuring the reproductive costs incurred by a parent providing for offspring, and more often than not people fail to distinguish it clearly from parental input (Clutton-Brock, 1984, 1991; Evans, 1990). A strong positive correlation between parental input and investment is therefore, in reality, rarely proven (Clutton-Brock, 1984; Evans, 1990). While it is thus possible that the decline in maternal responsiveness with age in our sows could be an indicator of reduced maternal investment contrary to predictions from the Terminal Investment Hypothesis, this would be on the assumption of a strong positive correlation between maternal responsiveness and maternal investment in sows.

(b) Evans (1990) suggests that parental input and investment can sometimes be inversely related, such that optimum parental input will decrease if reproductive costs (as determining parental investment) increase. Our study might provide an example of this. It could be, for example, that sows get less efficient at converting food into energy as they get older, which would increase the reproductive costs of providing for the offspring (e.g. nursing them for $4 \mathrm{~min}$ ). This would mean that maternal investment in domestic pigs increases with age, at least in outdoor pigs under commercial conditions, but that this is reflected in a lowering of parental input.

Thus, our observed decrease of maternal input with age can either be in line with predictions from Parental Investment Theory (b) or not (a). The interpretation of our results 
in terms of Parental Investment Theory has thrown up these two interesting possibilities, but has to remain speculative without knowledge of the precise relationship between maternal input and investment.

The absence of a relationship between responsiveness in the Piglet Scream Test and piglet mortality casts some doubt on whether responses in this test are useful measures of mothering ability, at least in outdoor sows kept under commercial conditions. Wechsler and Hegglin (1997) showed a relationship between the percentage of crushed piglets and responsiveness to playback of a piglet scream in sows kept in a loose farrowing pen without accounting for differences in responsiveness to the control sound. More recently, Andersen et al. (2005) have similarly demonstrated that indoor sows which never crushed any piglets responded more quickly to piglet distress calls on Day 7 or 8 than sows that had crushed several piglets. Śpinka et al. (2000), on the other hand, found no association between the responses in the Piglet Scream Test of primiparous sows kept in farrowing pens and overall piglet mortality between Day 0 and 4. Similarly, Grandinson et al. (2003) failed to find a phenotypic relationship between responses in the Piglet Scream Test and piglet mortality in the first days of lactation (D4 on average) in nine Swedish breeding herds. These and the present study all used different sow genotypes, which may go some way to explaining the different findings. Differences in the acoustic characteristics of the recorded piglet calls, and in particular the general lack of procedural standardization in the Piglet Scream Test, may also account for some of the variation between different studies. Furthermore, only the original study by Wechsler and Hegglin (1997) identified the cause of death in live-born piglets, and was thus able to identify how many piglets were actually killed by crushing. Without post-mortem analysis or a record of the events leading up to a piglet's death, it is difficult to accurately attribute cause of death (Edwards, 2002). Gross, non-specified mortality data, as were used in this study, may thus hide an underlying relationship between maternal responsiveness and crushing. In addition, our study used piglet mortality over the whole lactation period, which inevitably includes a greater number of deaths that are not caused by crushing than mortality estimates earlier in lactation. The sample size was also lower than originally planned because of restricted access to the farm during the Foot and Mouth Epidemic. This negatively affected the power of our study. Nonetheless, our study provides some first indication that in outdoor sows on commercial farms at least, maternal responsiveness as measured in the Piglet Scream and Piglet Defence Tests may not be related to gross pre-weaning mortality. It therefore cautions against the use of the Piglet Scream Test as a method for identifying (highly maternal) outdoor sows with good piglet survival in the absence of further evidence from a larger sample of sows and detailed post-mortem records. We are currently investigating whether the responses of commercial outdoor sows in the Piglet Scream and Defence Tests are associated with more subtle signs of offspring health and welfare, such as growth rates and handleability.

As previous studies on indoor sows (e.g. Hutson et al., 1991; Wechsler and Hegglin, 1997; Špinka et al., 2000; Pitts et al., 2002) we found large individual variation in the maternal responsiveness of outdoor sows. Hutson et al. (1991) had reported that about $60 \%$ of their 65 sows were responding while the remainder ignored the piglet stimulus. Sows had been kept in farrowing crates and parities ranged from first to ninth, with a median of fourth (Hutson et al., 1991). Similarly, Wechsler and Hegglin (1997) reported that only 
$63.6 \%$ of the 11 multiparous sows in their study changed posture during at least two of the three playbacks of a piglet scream. Probably because of habituation during the three repeats, only $42.6 \%$ of the sows in our study responded to a piglet scream by sitting up or standing up in at least two of the three playback repeats in the first parity, $34.6 \%$ in the second or third and $36.5 \%$ in the fourth. However, in none of the parities did responses to the piglet scream show the 'all-or-nothing' frequency distribution suggested by Hutson et al. (1991) such that sows might be identified as responders or non-responders (see Fig. 1). The fact that our animals habituated over repeated playbacks within $100 \mathrm{~min}$ in the Piglet Scream Test has implications for using the test in farrowing systems where sows are housed at high densities, such as farrowing crates or indoor loose housing systems. Repeated testing within hearing range of a sow may habituate her to the piglet scream before she herself is tested.

Our results provide some first evidence that outdoor sows remain consistent relative to each other across several parities such that the most responsive stay the most responsive even if overall responsiveness levels drop. Response strength in the Piglet Scream Test was correlated between the sows' first and second or third parities. It also tended to be correlated between second/third and fourth parities in two-tailed tests in spite of the small sample size. Sows were also consistent relative to each other between second/third and fourth parities in their defensiveness scores. Responses in the two tests only significantly co-varied in the sows' first parities, that is, at the same age and maternal experience as reported by Špinka et al. (2000) whose study looked at primiparous sows. Their Human-InThe-Nest Test elicited responses to a potential threat to the sows' piglets as did our Piglet Defence Test. Responses in their test were correlated to responsiveness to playback of a squeezed piglet on Day 2, and to playback of various piglet screams later on (Špinka et al., 2000). This led Špinka et al. (2000) to suggest 'protectiveness' as one of three potential behavioural characteristics determining maternal behaviour in pigs. For 'protectiveness', i.e. correlated responses in a Piglet Defence Test and the Piglet Scream Test, to be a stable maternal behavioural characteristic, responses in the two tests need to be demonstrated to remain correlated in further parities, and differences between individuals in this behavioural trait to be consistent across several parities. Our work to date suggests that responses in such two test do not necessarily remain correlated beyond the sows' first litters, and that 'protectiveness' sensu Špinka et al. (2000) may not be a stable individual behavioural characteristic of sows, at least not under the conditions of our study. Our findings also cautions against the generalisability of relationships found in gilts or primiparous sows to later maternal behaviour. To investigate this further, future work will examine whether the responses of outdoor sows in these tests of maternal responsiveness consistently co-vary with measures of spontaneous maternal behaviour over several parities, such as nursing frequencies, time spent away from the litter or interactions with the piglets.

\section{Acknowledgements}

This study was funded by the BBSRC of the UK through research grant 7/D11141 and a David Phillips Research Fellowship to G.M. We thank David Wilkinson and Debbie 


\section{Challinor of Downland Pigs for their dedicated co-operation and two anonymous referees} for their comments.

\section{References}

Andersen, I.L., Berg, S., Bøe, K.E., 2005. Crushing of piglets by the mother sow (Sus scrofa)—purely accidental or a poor mother. Appl. Anim. Behav. Sci. 93, 229-243.

Clutton-Brock, T.H., 1984. Reproductive effort and terminal investment in interoparous animals. Am. Nat. 132, 212-229.

Clutton-Brock, T.H., 1991. The Evolution of Parental Care. Princeton University Press, Princeton, NJ.

Cronin, G.M., Cropley, J.A., 1991. The effect of piglet stimuli on the posture changing behaviour of recently farrowed sows. Appl. Anim. Behav. Sci. 30, 167-172.

Edwards, S.A., 2002. Perinatal mortality in the pig: environmental or physiological solutions? Livestock Prod. Sci. 78, 3-12.

Edwards, S.A., Smith, W.J., Fordyce, C., Macmenemy, F., 1994. An analysis of the causes of piglet mortality in a breeding herd kept outdoors. Vet. Rec. 135, 324-327.

Evans, R.M., 1990. The relationship between parental input and investment. Anim. Behav. 39, 797-813.

Fleming, A.S., Morgan, H.D., Walsh, C., 1996. Experiential factors in postpartum regulation of maternal care. Adv. Study Behav. 25, 295-333.

Grafen, A., Hails, R., 2002. Modern Statistics for the Life Sciences. Oxford University Press, Oxford, p 351.

Grandinson, K., Rydhmer, L., Strandberg, E., Thodberg, K., 2003. Genertic analysis of on-farm tests of maternal behaviour in sows. Livestock Prod. Sci. 83, 141-151.

Graves, H.B., 1984. Behavior and ecology of wild and feral swine (Sus Scrofa). J. Anim. Sci. 58, 482-492.

Herskin, M.S., Jensen, K.H., Thodberg, K., 1998. Influence of environmental on maternal behaviour related to bonding, reactivity and crushing of piglets in domestic sows. Appl. Anim. Behav. Sci. 58, 241-254.

Hutson, G.D., Wilkinson, J.L., Luxford, B.G., 1991. The response of lactating sows to tactile, visual and auditory stimuli associated with a model piglet. Appl. Anim. Behav. Sci. 32, 129-137.

Hutson, G.D., Argent, M.F., Dickenson, L.G., Luxford, B.G., 1992. Influence of parity and time since parturition on responsiveness of sows to a piglet distress call. Appl. Anim. Behav. Sci. 34, 303-313.

Hutson, G.D., Price, E.O., Dickenson, L.G., 1993. The effect of playback volume and duration on the response of sows to piglet distress calls. Appl. Anim. Behav. Sci. 37, 31-37.

Marchant, J.N., Broom, D.M., Corning, S., 2001. The influence of sow behaviour on piglet mortality due to crushing in an open farrowing system. Br. Soc. Anim. Sci. 72, 19-28.

Pitts, A.D., Weary, D.M., Fraser, D., Pajor, E.A., Kramer, D.L., 2002. Alternative housing for sows and litters. Part 5. Individual difference in the maternal behaviour of sows. Appl. Anim. Behav. Sci. 291-306.

Riart, G.R., Edwards, S.A., English, P.R., 2000. Estudio de los factores que afectan mortalidad pre-destete en lechones nacidos a campo: compración con sistemas intensivos. In: Congreso Mercosur de Producción Porcina.

Signoret, J.P., Baldwin, B.A., Fraser, D., Hafz, E.S.E., 1975. The behaviour of swine. In: Hafez, E.S.E. (Ed.), The Behaviour of Domestic Animals. Balliere Tindall, London, pp. 295-329.

Špinka, M., Illmann, G., de Jonge, F., Andersson, M., Schuurman, T., Jensen, P., 2000. Dimensions of maternal behaviour characteristics in domestic and wild $\times$ domestic crossbred sows. Appl. Anim. Behav. Sci. 70, 99114.

Svendsen, J., Bengtsson, A.C., Svendsen, L.S., 1986. Occurrence and causes of traumatic injuries inneonatal pigs. Pig News Inf. 7, 159-170.

Thodberg, K., Jensen, K.H., Herskin, M.S., 2002. Nursing behaviour, postpartum activity and reactivity in sows: effect of farrowing environment, previous experience and temperament. Appl. Anim. Behav. Sci. 77, 53-76.

Trivers, R.L., 1972. Parental investment and sexual selection. In: Campbell, B. (Ed.), Sexual Selection and the Descent of Man. Aldine Press, Chicago, IL, pp. 136-170.

Vieuille, C., Berger, F., Le Pape, G., Bellanger, D., 2003. Sow behaviour involved in the crushing of piglets in outdoor farrowing huts-a brief report. Appl. Anim. Behav. Sci. 80, 109-115. 
Weary, D.M., Pajor, E.A., Fraser, D., Hokanen, A.M., 1996a. Sow body movements that crush piglets: a comparison between two types of farrowing accommodation. Appl. Anim. Behav. Sci. 49, 149-158.

Weary, D.M., Pajor, E.A., Thompson, D.K., Fraser, D., 1996b. The relationship between piglet body condition and proximity to the sow: a trade off between feeding and the risk of mortality by maternal crushing? Anim. Behav. 51, 619-624.

Wechsler, B., Hegglin, D., 1997. Individual difference in the behaviour of sows at the nest-site and crushing of piglets. Appl. Anim. Behav. Sci. 51, 39-49.

Wells, G.A.H., 1978. Post mortem techniques for the pig. Pig J. 2, 19-25.

Williams, G.C., 1966. Adaptation and Natural Selection: A Critique of Some Current Evolutionary Thought. Princeton University Press, Princeton, NY. 